Review began 01/04/2022 Review ended 01/05/2022 Published 01/09/2022

(c) Copyright 2022

Vardhan et al. This is an open access article distributed under the terms of the Creative Commons Attribution License CCBY 4.0., which permits unrestricted use, distribution, and reproduction in any medium, provided the original author and source are credited.

\section{Effect of Dance Movement Therapy on Cancer- Related Fatigue in Breast Cancer Patients Undergoing Radiation Therapy: A Pre-post Intervention Study}

Vishnu Vardhan ${ }^{1}$, Chanan Goyal ${ }^{2,3}$, Jaimini Chaudhari ${ }^{4}$, Vandana Jain ${ }^{5}$, Chaitanya A. Kulkarni ${ }^{6}$, Moli Jain ${ }^{1}$

1. Cardiorespiratory Physiotherapy, Datta Meghe Institute of Medical Sciences, Wardha, IND 2. Paediatric Physiotherapy, Datta Meghe Institute of Medical Sciences, Wardha, IND 3. Paediatric Physiotherapy, Government Physiotherapy College, Raipur, IND 4. Physiotherapy, Pravara Institute of Medical Sciences, Ahmednagar, IND 5. Radiation Oncology, Pravara Institute of Medical Sciences, Ahmednagar, IND 6. Community Physiotherapy, Datta Meghe Institute of Medical Sciences, Wardha, IND

Corresponding author: Chanan Goyal, chanangupta@gmail.com

\section{Abstract}

\section{Introduction}

Dance movement therapy (DMT) is a movement-based psychosocial intervention that incorporates the therapeutic components of dance movements and group psychotherapy. DMT, also known as creative movement therapy (CMT) is a psychotherapy used as a complementary therapy in cancer care. It helps in enhancing mood, emotions, self-expression and helps to rebuild self-confidence. Besides, it allows the patients to recognise their own strengths and weaknesses as well as helps to improve physical capabilities.

\section{Methods}

By simple random sampling method, 30 breast cancer patients were recruited at Pravara Rural Hospital, Loni, Maharashtra, India. The participants were in the age range of 30-60 years based on the inclusion and exclusion criteria. Pre-intervention scores of cancer-related fatigue (CRF) were taken using the Brief Fatigue Inventory (BFI) scale and intervention was given for 45 minutes each day for 5 days a week, over a span of 2 weeks. Thereafter, post-intervention assessment was done and the scores were noted. Pre-intervention and post-intervention scores were compared using paired t-test.

\section{Results}

The mean and standard deviation (SD) of pre- and post-BFI scores derived by using paired t-test was 73.76 (8.6) and 69.33 (9.8), respectively, with a p-value of $<0.001$, which is highly significant.

\section{Conclusion}

The results of the present study revealed that DMT seems to be effective in reducing some amount of CRF in breast cancer patients undergoing radiation therapy. Besides, it turned out to be an engaging, entertaining and cost-effective approach. The investigation showed that DMT appears to be beneficial in reducing the side effects of radiation therapy such as pain, stress, anxiety and fear, giving a psychotherapeutic relief but did not completely remove the persistent fatigue experienced by the breast cancer patients. Thus, further investigation with long-term follow-up is recommended.

Categories: Physical Medicine \& Rehabilitation, Radiation Oncology, Therapeutics

Keywords: cancer-related fatigue, physiotherapy, radiation therapy, creative movement therapy, dance movement therapy, brief fatigue inventory, breast cancer

\section{Introduction}

Fatigue is characterised as a subjective sense of tiredness or inability to do certain tasks due to extreme weakness, which is a common and distressing symptom usually associated with cancer or cancer treatment and is termed as cancer-related fatigue (CRF). CRF differs from the fatigue experienced by a healthy individual because CRF is non-transient and is less likely to be relieved by rest as cancer patients experience constant fatigue [1]. CRF can be defined as "a painful, obstinate, subjective sense of physical, emotional and/or cognitive weakness or exhaustion related to cancer treatment that is not proportional to recent physical activity and interferes with usual functioning” [2]. Cancer patients reported having CRF from the time of diagnosis, throughout the treatment and even months or years after the completion of treatment [3]. CRF can affect the patient on multiple levels of psychological and physical functioning and usually causes a noticeable decrease in the patient's quality of life [4]. 
Adjuvant radiation therapy (RT) is used to decrease the risk of relapse in women with breast cancer. Patients undertaking RT often experience multiple symptoms including pain, fatigue, stress, and sleep disturbance, with reported occurrence rates of over $60 \%$ near the end of RT. A large volume of research has found the usefulness of non-pharmacological interventions to alleviate treatment-related symptoms in cancer patients, use of physical movements along with the ongoing RT to relieve pain and to reduce CRF [5].

Dance movement therapy (DMT) is a movement-based psychosocial intervention that integrates the therapeutic components of dance movement and group psychotherapy. It empowers the patients to enhance self-expression, accept and reconnect with their own bodies, cope with feelings of depression and fear, rebuild the shattered self-confidence, and strengthen personal resources. The group approach permits them to share their emotions and concerns, and also coping strategies with others [6,7]. DMT can be defined as the therapeutic use of movements that further enhances emotional, cognitive, and physical and the social integration of the individual [8].

Application of DMT in group therapy helps to reduce the CRF in breast cancer patients [9]. Previous research showed that a brief DMT intervention over the course of RT would benefit the patients by buffering their treatment-related symptoms while the symptoms would worsen in women who did not receive DMT [10,11]. Studies revealed physical activity along with adjuvant therapies (RT) improves the cancer patients' overall condition but there is a gap in understanding the reduction of CRF with the help of low-impact physical activity like DMT. The effects of DMT on patients receiving active cancer treatment are yet to be studied. More studies are needed to elucidate the effects of DMT on physical and psychological outcomes in cancer patients [12]. Active participation and movement-based physiotherapy have been observed to be beneficial in a variety of other common and rare disorders [13-15].

\section{Materials And Methods}

Prior to the commencement, the study was approved by the Institutional Review Board known as Institutional Ethical Committee of Dr. A.P.J. Abdul Kalam College of Physiotherapy at Pravara Institute of Medical Sciences with approval number BPT/INT/2019/05. Consent from the department of oncology and RT of Pravara Rural Hospital, Loni, India was also taken. Detailed information regarding the nature of study was given to the patients before participation. Written consent forms were taken from the patients and permissions were obtained. By simple randomised sampling, 30 breast cancer patients were recruited. Inclusion criteria included radical mastectomy patients undergoing RT for breast cancer between the age range of 30 and 60 years, having stage of cancer one to three with BFI score above 45 who were willing to participate in the study. Those patients fulfilling the inclusion criteria were requested to participate in the study. Subjects who had recent history of trauma, pregnant patients, patients with history of sleep apnoea or anaemia and the patients for whom exercise was contraindicated were excluded from the study. All the patients were given the Brief Fatigue Inventory (BFI) and were asked to fill the questionnaire, as a preintervention assessment and their scores were taken. BFI has been included in the Appendices section. Participants were informed that the use of the collected data is purely for the purpose of the study.

\section{Outcome measure}

BFI was the scale that was used to assess the severity of fatigue related to cancer, before and after the intervention. It is a valid and reliable scale to measure fatigue in patients with various chronic illnesses including cancer $[16,17]$.

\section{Procedure}

The participants, in addition to the ongoing treatment, were given group therapy in the form of DMT. Each session lasted for 45 minutes and one such session was imparted on 5 days of a week for a duration of 2 weeks. Each session was divided into three sections namely warm-up phase, development phase and cooldown phase. Warm-up phase included introducing and greeting each other with welcoming gestures and breathing exercises for 5 minutes. Development phase is the most crucial because it challenges the patients to perform all the movements to the best of their abilities for around 30 minutes. As this study was conducted in a rural medical hospital, regional music was used allowing the patients to enjoy the rhythm. The patients were allowed to move and dance according to their comfort while following the steps as demonstrated by the physiotherapist. Cool-down phase was for 10 minutes which included sitting in circle holding hands and doing deep breathing exercises followed by meditation or sitting in silence. Last, everyone was allowed to share what they felt during the whole session.

\section{Data collection}

The outcome measure, BFI, is a nine-item, 11-point rating scale developed to assess subjective fatigue. The first three questions measure fatigue severity from 0 which indicates "no fatigue" to 10 which indicates "worst levels of fatigue." The following six questions assess fatigue interference with daily activities including general activity, mood, walking ability, normal work (both inside and outside the home), relations with other people and enjoyment of life. Response options range from 0 , indicating "does not interfere," to 10 indicating, "completely interferes.” Higher scores on the BFI correspond to greater self-reported levels of fatigue. The participant has to indicate by circling how much fatigue they feel from 0 to 10 while doing some 


\section{Cureus}

activity or during some situation. The participants were asked to fill this questionnaire before and after the 2 weeks' sessions. The maximum score in BFI is 90 . Table 1 shows the categorisation of fatigue according to the BFI score.

Fatigue Category

Mild

Moderate

Severe

\section{BFI Score}

$30-45$

$45-75$

$75-90$

TABLE 1: Fatigue categories according to BFI scores

BFI: Brief Fatigue Inventory

\section{Results}

A total of 30 participants were taken according to the selection criteria. Baseline characteristics (age and pre-interventional BFI scores) were taken and analysed. The mean age ( $\left.{ }^{ \pm} \mathrm{SD}\right)$ was $45.66 \pm 8.83$. The preintervention data collected showed the average $( \pm \mathrm{SD})$ of $73.76 \pm 8.6$ while the post-intervention data collected showed the average $( \pm \mathrm{SD})$ of $69.33+9.8$ as depicted in Figure 1 .

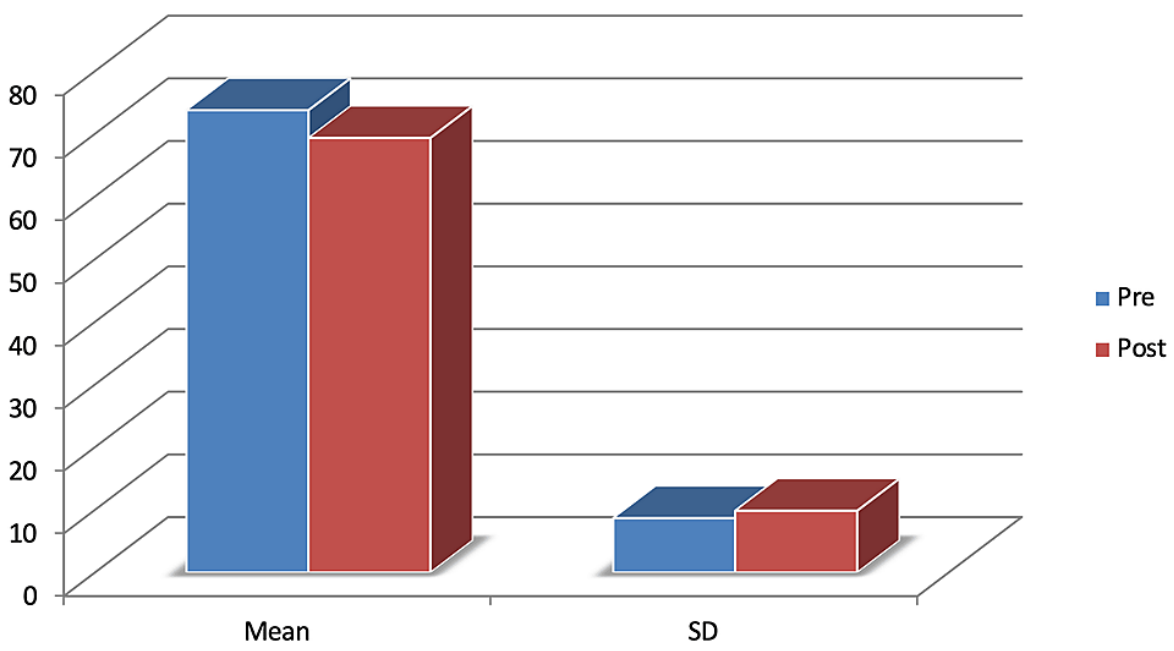

FIGURE 1: Graph showing pre- and post-intervention mean and standard deviation of BFI scores

BFI: Brief Fatigue Inventory

The comparison of pre- and post-intervention BFI scores was found to be statistically significant using the paired t-test in which $\mathrm{p}$-value was $0.001(\mathrm{p}>0.05)$ which is highly significant and t value was 2.62 as shown in Table 2. 


\section{Cureus}

\begin{tabular}{|c|c|c|c|c|}
\hline & Pre & Post & Student's paired t-test & p-value and significance \\
\hline Mean & $73.76+8.6$ & $69.33+9.8$ & \multirow{2}{*}{2.62} & \multirow{2}{*}{$p=0.001$, highly significant } \\
\hline SD & 8.67 & 9.85 & & \\
\hline
\end{tabular}

TABLE 2: Pre- and post-intervention mean and standard deviation of BFI scores; $t$-value and pvalue after performing paired t-test

BFI: Brief Fatigue Intervention

\section{Discussion}

The effect of DMT has been studied in a variety of conditions including depression, dementia and cancer [18-21]. DMT has been investigated for cancer care, in both, children and adult population. Paediatric medical DMT is an approach that emphasises the expression of emotions through dance and movement [22]. Besides, different forms of dance are under consideration for therapeutic use for breast cancer patients with CRF [23,24]. Also, apart from group therapy, DMT has been utilised as an individualised therapy in a few studies $[7,25]$. Outcome measures and parameters used in various studies on use of DMT in patients with cancer included quality of life, anxiety, stress, pain, fatigue, vitality, body awareness, self-care and selfefficacy apart from similar others [7]. Fatigue is associated with cancer as well as with adjuvant cancer treatments like chemotherapy and radiotherapy but the pathogenesis of fatigue is largely unknown [26]. CRF is a frequently seen yet under-diagnosed phenomenon in patients with cancer. The currently available evidence shows that well-designed exercise is effective in alleviating CRF [27]. In breast cancer patients, fatigue was found to be one of the major limiting factors in return to work [28]. The results of previous studies were inconsistent and further investigations with larger sample sizes have been warranted in current literature.

The present study aims to identify the effect of DMT on breast cancer patients with persistent CRF which is a common side effect of RT. The purpose of DMT is to help people to achieve self-awareness and to come in contact with conscious and unconscious parts of one's own personality during each session. It demonstrated significant improvement in mood after each DMT session. There was a decreased sense of fear, improvement in the overall attitude and feeling of joy which helped them to fight the chronic disease with self-confidence [29].

The results of the present study showed that DMT seems to be effective in reducing fatigue up to a certain level $(\mathrm{t}=2.62, \mathrm{p}<0.001)$ which is in accordance with the study conducted by Ho et al., who investigated DMT during RT and demonstrated significant improvement in stress, pain and fatigue. The study stated that there was potential clinical value of DMT in pain reduction and DMT could be incorporated as part of an integrative cancer care as prophylaxis at the start of RT. Although Ho et al. reported a large treatment effect of DMT on pain severity reduction, our DMT group showed no significant effect on improvement of quality of life $[30,31]$. The lack of significant findings could be attributed to the short duration (2 weeks) of the intervention. Given the time constraint in relation to the RT which is given for 5 weeks, dramatic changes in quality of life or emotional changes were not expected. There was a significant decrease in fatigue after three to four sessions but reoccurred after the gap of 2 days in a week as subjectively reported by the participants. The patients complained of obstinate fatigue after the RT. Hence, the preferable time for the intervention was in the morning hours [32].

The limitations of the study included the recruitment of only ambulatory patients. Thus, the result cannot be generalised to those patients who were less mobile. Patients who were in severe pain and had severe side effects of nausea and vomiting were not included in the program. They lacked motivation or stamina to participate in the program. This may lead to sampling bias to the participants who were not able to participate, although simple random sampling method was used. In general, the elder age group had more pain than the younger [33].

Dance represents a psychotherapeutic treatment and a form of physical activity, based on body awareness, expression and acceptance, in order to facilitate the physical, emotional, cognitive and spiritual integration, thus, making it a holistic approach for cancer care. DMT has shown promising initial result in improving quality of life and mental health of patients with breast cancer [7,34]. It is possible to remark that DMT seems to be helpful in reducing CRF up to a certain level by improving the mental and emotional status of breast cancer patients but further studies are required [21].

\section{Conclusions}

The result of the present study revealed that DMT seems to be effective in reducing some amount of CRF in 


\section{Cureus}

breast cancer patients undergoing RT. The intervention given for 2 weeks for 5 days per week showed that DMT can be helpful in reducing the side effects of RT such as pain, stress, anxiety and fear, giving a psychotherapeutic relief but did not completely reduce the persistent fatigue experienced by the breast cancer patients. It is engaging, entertaining and cost-effective at the same time. DMT with different dance forms in context of culture can be considered as a complementary therapy for patients with various types of cancer in all age groups. This study opens up avenues to explore physiotherapeutic movements in the management of CRF warranting further investigation with larger sample size and long-term follow-up.

\section{Appendices}

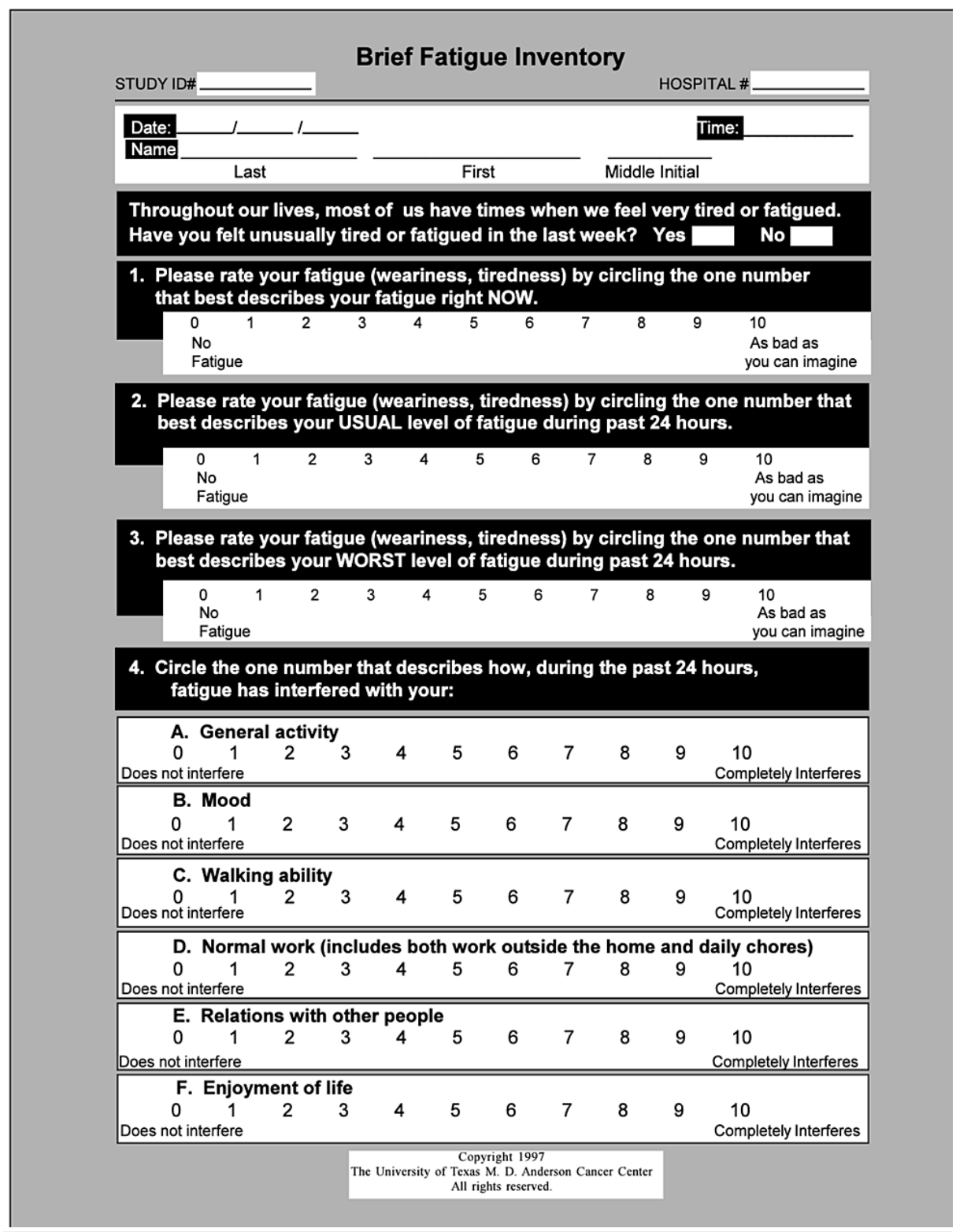

FIGURE 2: Brief Fatigue Inventory

\section{Additional Information}

\section{Disclosures}

Human subjects: Consent was obtained or waived by all participants in this study. Institutional Ethical Committee of Pravara Institute of Medical Sciences issued approval BPT/INT/2019/05. The committee approved the research. Animal subjects: All authors have confirmed that this study did not involve animal subjects or tissue. Conflicts of interest: In compliance with the ICMJE uniform disclosure form, all authors declare the following: Payment/services info: All authors have declared that no financial support was received from any organization for the submitted work. Financial relationships: All authors have declared that they have no financial relationships at present or within the previous three years with any organizations that might have an interest in the submitted work. Other relationships: All authors have 
declared that there are no other relationships or activities that could appear to have influenced the submitted work.

\section{References}

1. Banipal RPS, Singh H, Singh B: Assessment of cancer-related fatigue among cancer patients receiving various therapies: a cross-sectional observational study. Indian J Palliat Care. 2017, 23:207-11. 10.4103/IJPC.IJPC_135_16

2. Kröz M, Reif M, Glinz A, et al.: Impact of a combined multimodal-aerobic and multimodal intervention compared to standard aerobic treatment in breast cancer survivors with chronic cancer-related fatigue results of a three-armed pragmatic trial in a comprehensive cohort design. BMC Cancer. 2017, 17:166. 10.1186/s12885-017-3142-7

3. Kessels E, Husson O, van der Feltz-Cornelis CM: The effect of exercise on cancer-related fatigue in cancer survivors: a systematic review and meta-analysis. Neuropsychiatr Dis Treat. 2018, 14:479-94. 10.2147/NDT.S150464

4. Olsson Möller U, Beck I, Rydén L, Malmström M: A comprehensive approach to rehabilitation interventions following breast cancer treatment - a systematic review of systematic reviews. BMC Cancer. 2019, 19:472. 10.1186/s12885-019-5648-7

5. Ho RTH, Fong TCT, Cheung IKM, Yip PSF, Luk M-Y: Effects of a short-term dance movement therapy program on symptoms and stress in patients with breast cancer undergoing radiotherapy: a randomized, controlled, single-blind trial. J Pain Symptom Manage. 2016, 51:824-31. 10.1016/j.jpainsymman.2015.12.332

6. Yadav V, Naqvi WM, Burhani T: Pandemics and physiotherapy: an overview of the role of the physiotherapists in restoring functions and quality of life. Int J Res Pharm Sci. 2020, 11:1898-901. 10.26452/ijrps.v11iSPL1.4550

7. Goodill SW: Accumulating evidence for dance/movement therapy in cancer care . Front Psychol. 2018, 9:1778. 10.3389/fpsyg. 2018.01778

8. Ritter M, Low KG: Effects of dance/movement therapy: a meta-analysis. Arts Psychother. 1996, 23:249-60. 10.1016/0197-4556(96)00027-5

9. Carminatti M, Boing L, Leite B, et al.: Effects of belly dancing on body image and self-esteem in women with breast cancer - pilot study. Rev Bras de Med Esporte. 2019, 25:464-68. 10.1590/1517-869220192506220067

10. Ho RTH, Fong TCT, Yip PSF: Perceived stress moderates the effects of a randomized trial of dance movement therapy on diurnal cortisol slopes in breast cancer patients. Psychoneuroendocrinology. 2018, 87:119-26. 10.1016/j.psyneuen.2017.10.012

11. Saifee SS, Yadav V, Jain M, Kulkarni CA, Naqvi WM: A comprehensive pulmonary rehabilitation program for the management of post- tuberculosis pneumothorax: a case study. J Med Pharm Allied Sci. 2021, 10:2900-3. 10.22270/jmpas.V10I3.1127

12. Aktas G, Ogce F: Dance as a therapy for cancer prevention . Asian Pac J Cancer Prev. 2005, 6:408-11.

13. Goyal C, Goyal V, Naqvi WM: Goyal-Naqvi Syndrome (concurrent trisomy 10p and terminal 14q deletion): a review of the literature. Cureus. 2021, 13:e16652. 10.7759/cureus.16652

14. Goyal C, Naqvi WM, Sahu A, Aujla AS: Xia-Gibbs Syndrome: a review of literature . Cureus. 2020, 12:e12352. 10.7759/cureus. 12352

15. Goyal C, Naqvi WM, Sahu A: An atypical case of febrile infection-related epilepsy syndrome following acute encephalitis: impact of physiotherapy in regaining locomotor abilities in a patient with neuroregression. Pan Afr Med J. 2020, 36:101. 10.11604/pamj.2020.36.101.23855

16. Chen YW, Coxson HO, Reid WD: Reliability and validity of the brief fatigue inventory and dyspnea inventory in people with chronic obstructive pulmonary disease. J Pain Symptom Manage. 2016, 52:298-304. 10.1016/j.jpainsymman.2016.02.018

17. Paramita N, Nusdwinuringtyas N, Nuhonni SA, Atmakusuma TD, Ismail RI, Mendoza TR, Cleeland CS: Validity and reliability of the Indonesian version of the Brief Fatigue Inventory in cancer patients . J Pain Symptom Manage. 2016, 52:744-51. 10.1016/j.jpainsymman.2016.04.011

18. Meekums B, Karkou V, Nelson EA: Dance movement therapy for depression. Cochrane Database Syst Rev. 2015, CD009895. 10.1002/14651858.CD009895.pub2

19. Karkou V, Meekums B: Dance movement therapy for dementia. Cochrane Database Syst Rev. 2017, 2:CD011022. 10.1002/14651858.CD011022.pub2

20. Millman LS, Terhune DB, Hunter EC, Orgs G: Towards a neurocognitive approach to dance movement therapy for mental health: a systematic review. Clin Psychol Psychother. 2021, 28:24-38. 10.1002/cpp.2490

21. Bradt J, Shim M, Goodill SW: Dance/movement therapy for improving psychological and physical outcomes in cancer patients. Cochrane Database Syst Rev. 2015, CD007103. 10.1002/14651858.CD007103.pub3

22. Tortora S: Children are born to dance! Pediatric medical dance/movement therapy: the view from integrative pediatric oncology. Children (Basel). 2019, 6:14. 10.3390/children6010014

23. Oei SL, Rieser T, Becker S, Groß J, Matthes H, Schad F, Thronicke A: TANGO: effect of tango Argentino on cancer-associated fatigue in breast cancer patients-study protocol for a randomized controlled trial. Trials. 2021, 22:866. 10.1186/s13063-021-05869-3

24. Reis D, Walsh ME, Jones T, Young-McCaughan S: A review of Nia as an exercise option for cancer survivors . Clin J Oncol Nurs. 2014, 18:689-92. 10.1188/14.CJON.689-692

25. Madden JR, Mowry P, Gao D, Cullen PM, Foreman NK: Creative arts therapy improves quality of life for pediatric brain tumor patients receiving outpatient chemotherapy. J Pediatr Oncol Nurs. 2010, 27:133-45. $10.1177 / 1043454209355452$

26. Yang S, Chu S, Gao Y, Ai Q, Liu Y, Li X, Chen N: A narrative review of cancer-related fatigue (CRF) and its possible pathogenesis. Cells. 2019, 8:738. 10.3390/cells8070738

27. Thong MSY, van Noorden CJF, Steindorf K, Arndt V: Cancer-related fatigue: causes and current treatment options. Curr Treat Options Oncol. 2020, 21:17. 10.1007/s11864-020-0707-5

28. Schmidt ME, Scherer S, Wiskemann J, Steindorf K: Return to work after breast cancer: the role of treatmentrelated side effects and potential impact on quality of life. Eur J Cancer Care. 2019, 28:e13051. 


\section{Cureus}

10.1111/ecc. 13051

29. Savidaki M, Demirtoka S, Rodríguez-Jiménez R-M: Re-inhabiting one's body: a pilot study on the effects of dance movement therapy on body image and alexithymia in eating disorders. J Eat Disord. 2020, 8:22.

10.1186/s40337-020-00296-2

30. Koch SC, Riege RFF, Tisborn K, Biondo J, Martin L, Beelmann A: Effects of dance movement therapy and dance on health-related psychological outcomes. A meta-analysis update. Front Psychol. 2019, 10:1806. 10.3389/fpsyg.2019.01806

31. Ho RTH, Lo PHY, Luk MY: A good time to dance? A mixed-methods approach of the effects of dance movement therapy for breast cancer patients during and after radiotherapy. Cancer Nurs. 2016, 39:32-41. 10.1097/NCC.0000000000000237

32. Cummings GG, Lee SD, Tate KC: The evolution of oncology nursing: leading the path to change . Can Oncol Nurs J. 2018, 28:314-7.

33. Berger AM, Kuhn BR, Farr LA, Von Essen SG, Chamberlain J, Lynch JC, Agrawal S: One-year outcomes of a behavioral therapy intervention trial on sleep quality and cancer-related fatigue. J Clin Oncol. 2009, 27:6033-40. 10.1200/JCO.2008.20.8306

34. Fatkulina N, Hendrixson V, Rauckiene-Michealsson A, Kievisiene J, Razbadauskas A, Agostinis Sobrinho C: Dance/movement therapy as an intervention in breast cancer patients: a systematic review . Evid Based Complement Alternat Med. 2021, 2021:1-6. 10.1155/2021/4989282 